\title{
How I use Transcranial Doppler
}

\author{
Chiara Robba ${ }^{1}$ and Fabio Silvio Taccone ${ }^{2^{*}}$
}

Keywords: Transcranial Doppler, Flow velocity, Cerebral blood flow, Neurointensive care

\section{Introduction}

Transcranial Doppler (TCD) is a bedside, low-cost, and non-invasive technique able to evaluate cerebral hemodynamics [1]; the implementation of transcranial color-coded duplex sonography (TCCS) aids in evaluating the brain anatomy and intracranial lesions [2], real-time monitoring of "basic" (flow velocity (FV) and pulsatility index (PI)) as well as "advanced" TCD-derived parameters (Table 1; Fig. 1). In practice, we use a $2-\mathrm{MHz}$ probe, and most information is obtained by insonating the middle cerebral artery through the temporal window; other windows include the transorbital, occipital, and submandibular windows. TCCD has the advantage to provide a direct visualization of the cerebral anatomy vessels and allow angle correction to assess FV [2]. TCD/TCCD practice is part of the standard training in our institution, and examinations are routinely performed by the medical staff.

We discussed herein on how we use TCD in neurocritically ill patients for hemodynamic indications; some of these proposals could also be used in non-brain injured critically ill patients at a high risk of cerebral complications.

\section{Non-invasive assessment of intracranial pressure}

When the indications for invasive intracranial pressure (ICP) monitoring are met, we recommend intraparenchymal or intraventricular probes, as TCD cannot substitute invasive ICP measurement [3]. However, when indications are unclear or invasive methods are not available (i.e., low-income countries) or contraindicated (i.e., severe coagulopathy), we use TCD as a "triage" tool to non-invasively discriminate patients who are at risk of developing intracranial hypertension $[1,4,5]$. We do not rely on only PI (i.e., PI $>1.4$ ), because other conditions (Additional file 1: Table S1) could affect this parameter [2]. As such, after having considered these conditions,

\footnotetext{
* Correspondence: ftaccone@ulb.ac.be

${ }^{2}$ Department of Intensive Care Medicine, Erasme Hospital, Université Libre de Bruxelles (ULB), Route de Lennik, 808, 1070 Brussels, Belgium

Full list of author information is available at the end of the article
}

we use the combination of elevated PI and low diastolic FV $(<20 \mathrm{~cm} / \mathrm{s})$ to suggest elevated ICP at the bedside. Moreover, we also estimate ICP using formulas combining FV and blood pressure $[5,6]$, but only as "confirmatory" findings before additional validation of their accuracy will be available. Finally, we perform repeated TCD assessment rather than a single examination (i.e., every $1-2 \mathrm{~h}$ ) to better understand the changes in the brain hemodynamics following an increase in ICP or after specific ICP-directed therapies.

\section{Diagnosis of brain death}

Although the diagnosis of brain death is based on neurological examination, we use routinely TCD as an ancillary test to demonstrate the absence of cerebral blood flow (CBF) [7]. We use one of the following TCD patterns to determine "cerebral" circulatory arrest (CCA): reverberating flow, systolic spikes, and disappearance of previously recorded FV $[2,7]$. According to local practices, when we perform TCD and analyze the waveforms suggesting impending CCA, all the vessels of the circle of Willis through the trans-temporal and occipital windows are examined, as only the detection of the abovementioned flow patterns in all the major intracranial vessels is consistent for brain death [8]. When no intracranial signal is found but brain death criteria are met, we perform a brain CT perfusion or angiography to detect CCA.

\section{Cerebral autoregulation}

We assess cerebral autoregulation (CA) at the bedside as altered CA is related with a poor outcome in many diseases and may increase the risk of cerebral damage [9]. In case of impaired CA, we use TCD to target blood pressure to a level corresponding to the patient's individual optimal autoregulatory status. The most simple methods to assess $\mathrm{CA}$ at the bedside are (a) the static autoregulatory index [9], which is obtained by calculating the percentage of changes in cerebrovascular resistance $(\mathrm{CVR}=$ mean arterial pressure/mean FV) after changes in arterial blood 
Table 1 Common parameters derived from transcranial Doppler

\begin{tabular}{|c|c|c|c|c|c|c|}
\hline & Abbreviation or formula & Normal values & Elevated ICP & Brain death & Cerebral autoregulation & Cerebral vasospasm \\
\hline Pulsatility index & $\mathrm{PI}=(\mathrm{sFV}-\mathrm{dFV}) / \mathrm{mFV}$ & $<1.4$ & $>1.4$ & - & - & - \\
\hline Mean FV & $\mathrm{mFV}$ & $60-80 \mathrm{~cm} / \mathrm{s}[2]$ & - & - & - & $\begin{array}{l}\text { Mild } \geq 120 \mathrm{~cm} / \mathrm{s} \\
\text { Moderate }=120- \\
200 \mathrm{~cm} / \mathrm{s} \\
\text { Severe } \geq 200 \mathrm{~cm} / \mathrm{s} \\
\text { (with } L R<3 \text { ) }\end{array}$ \\
\hline Diastolic FV & $d F V$ & $>20 \mathrm{~cm} / \mathrm{s}$ & $<20 \mathrm{~cm} / \mathrm{s}$ & $\begin{array}{l}\text { Negative or } \\
\text { absent }\end{array}$ & - & Increased \\
\hline Mean flow index & Mx & $<0.3$ & $>0.3$ & - & $>0.3$ (impaired) & $>0.3$ \\
\hline Lindegaard ratio & $\begin{array}{l}\mathrm{LR}=\mathrm{mFV} \mathrm{MCA} / \mathrm{mFV} \\
\text { extracranial ICA }\end{array}$ & $<3$ & - & - & - & $\begin{array}{l}\text { Mild } \geq 3 \\
\text { Moderate }=3-6 \\
\text { Severe } \geq 6\end{array}$ \\
\hline THR test & & & & & $\begin{array}{l}\text { Less than } 10 \% \text { increase } \\
\text { from baseline sFV (impaired) }\end{array}$ & \\
\hline
\end{tabular}

FV flow velocity, MCA middle cerebral artery, ICA internal carotid artery, $M x$ mean flow index, $d F V$ diastolic flow velocity, $m F V$ mean flow velocity, $s F V$ systolic flow velocity, $C A$ cerebral autoregulation, $T H R$ transient hyperemic test

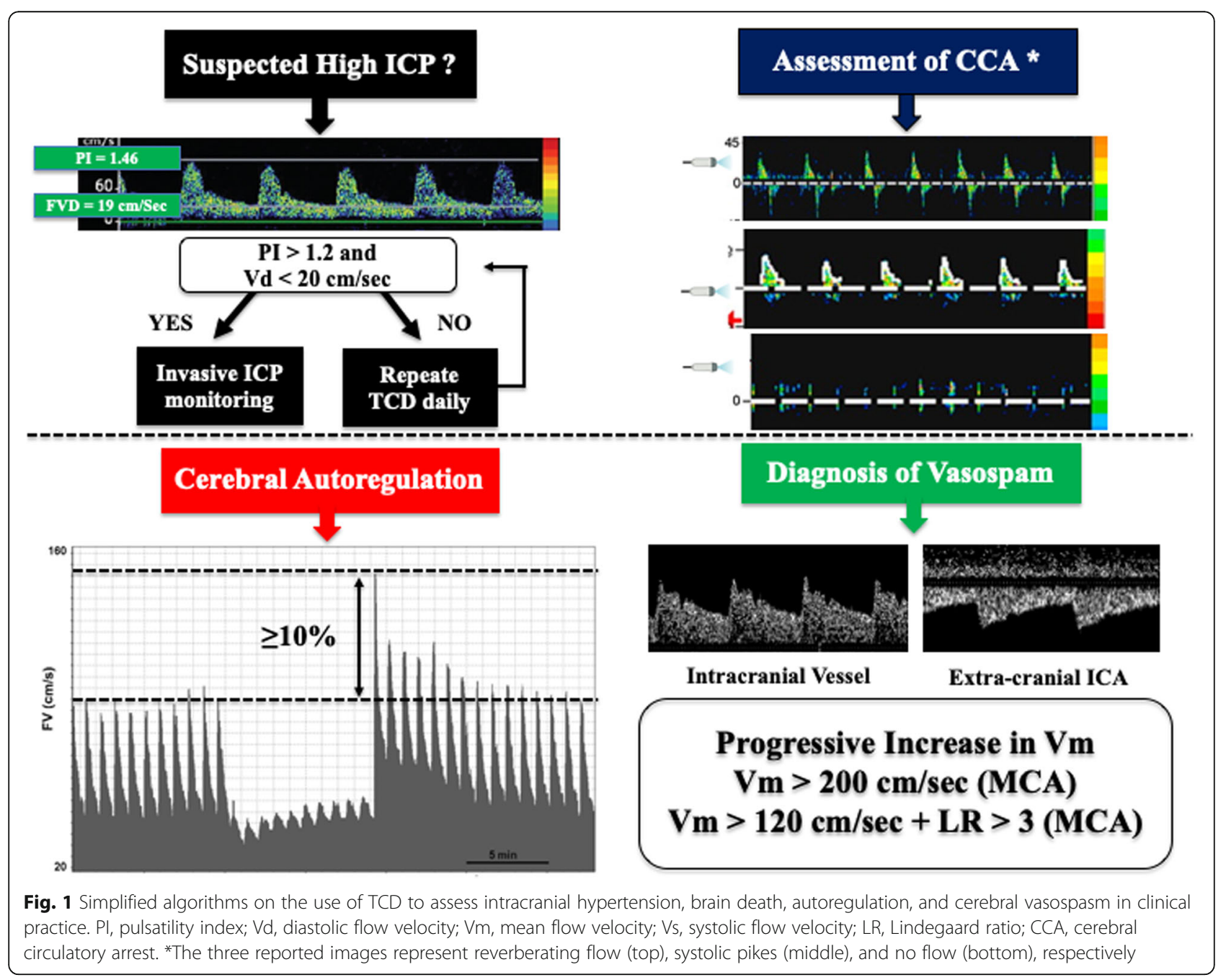


pressure, or (b) the transient hyperemic response test (if there are no risks of embolism or hemodynamic instability), which is obtained by compressing the carotid artery and calculating the percentage of change in systolic FV from the baseline (an increase $\geq 10 \%$ is considered as intact CA) [10]. Clinicians have to consider that the monitoring of dynamic autoregulation, using the mean flow index $(\mathrm{Mx})$, which is calculated as the correlation coefficient indices between FV and CPP during spontaneous fluctuations in blood pressure, would be more accurate to assess CA [11]. However, this method requires a specific software and a higher competency to interpret the data to improve patients' management.

\section{Cerebral vasospasm}

Detection of cerebral vasospasm following aneurysmal subarachnoid hemorrhage ( $\mathrm{SAH}$ ) is crucial as this is one of the main determinants of delayed cerebral ischemia and poor neurological outcome in this setting [12]. Although angiography remains the gold standard, we use TCD daily to assess vasospasm, to guide additional investigations, and to monitor the clinical treatment. Indeed, we evaluate the constriction of the cerebral vessels that is associated with a progressive increase of mean FV [13]. In daily practice, we perform serial TCD examinations (one to two/ day) in all SAH patients, together with close neurological clinical monitoring; we use TCD for the assessment of all main intracranial vessels and, using TCCD, investigate different segments of such vessels, as vasospasm could be extremely localized. In the presence of clinical suspicious of vasospasm (i.e., neurological deterioration), we use the cutoff of MCA mean flow velocity $(\mathrm{mFV})>200 \mathrm{~cm} / \mathrm{s}$ [14] to immediately initiate therapy and perform additional confirmatory imaging tests (i.e., cerebral CT perfusion or angiography). If $\mathrm{mFV}>120 \mathrm{~cm} / \mathrm{s}$ and $<200 \mathrm{~cm} / \mathrm{s}$, we assess the $\mathrm{mFV}$ in the extracranial internal carotid artery using the submandibular window and calculate the Lindegaard ratio (LR; Table 1) to differentiate vasospasm from cerebral hyperemia [15]. As TCD has a sensitivity of $90 \%$ (95\% confidence intervals [CIs] 77-96\%), specificity of $71 \%$ (95\% CI $51-84 \%$ ), positive predictive value of $57 \%$ (95\% CI $38-71 \%$ ), and negative predictive value of $92 \%$ (95\% CI 83-96\%) to diagnose vasospasm of MCA [13], we still perform cerebral CT perfusion or angiography in case of clinical suspicion of vasospasm with mFV below $<120$ $\mathrm{cm} / \mathrm{s}$. For other intracranial vessels, in the absence of validated mFV cutoffs, we combine clinical examination, repeated TCD showing a progressive increase in FV, and $\mathrm{CT}$ perfusion to detect vasospasm.

\section{Conclusions}

We often use TCD to monitor brain hemodynamics in critically ill patients. Future TCD development, such as the assessment of the compliance of arterial and cerebrospinal fluid compartment as well as critical capillary closing pressure, will further expand its use in this setting [1].

\section{Supplementary information}

Supplementary information accompanies this paper at https://doi.org/10. 1186/s13054-019-2700-6.

Additional file 1: Table S1. Factors that may influence pulsatility index (PI) and flow velocities. (DOCX 13 kb)

\section{Abbreviations}

CA: Cerebral autoregulation; CBF: Cerebral blood flow; CPP: Cerebral perfusion pressure; CVR: Cerebrovascular resistance; CT: Computed tomography; FV: Flow velocity; ICP: Intracranial pressure; LR: Lindegaard ratio; mFV: Mean flow velocity; Mx: Mean flow index; NIC: Neurointensive care; PI: Pulsatility index; SARI: Static autoregulatory index; sROR: Static rate of regulation; SAH: Subarachnoid hemorrhage; TCD: Transcranial Doppler; TBI: Traumatic brain injury

\section{Acknowledgements}

Not applicable

Authors' contribution

FST and CR conceived the study and drafted the present manuscript. Both authors read and approved the final manuscript.

Funding

No funding was provided for this study.

\section{Availability of data and materials \\ Not applicable}

Ethics approval and consent to participate Not applicable

\section{Consent for publication}

Not applicable

\section{Competing interests}

The authors declare that they have no competing interests for this manuscript.

\section{Author details}

${ }^{1}$ Policlinico San Martino, IRCCS per I'Oncologia e Neuroscienze, Dipartimento di Scienze Chirurgiche e Diagnostiche Integrate, Università degli Studi di Genova, Genova, Italy. ${ }^{2}$ Department of Intensive Care Medicine, Erasme Hospital, Université Libre de Bruxelles (ULB), Route de Lennik, 808, 1070 Brussels, Belgium.

Received: 26 September 2019 Accepted: 11 December 2019 Published online: 23 December 2019

\section{References}

1. Robba C, Cardim D, Sekhon M, Budohoski K, Czosnyka M. Transcranial Doppler: a stethoscope for the brain-neurocritical care use. J Neurosci Res. 2018;96:720-30.

2. Robba C, Goffi A, Geeraerts T, Cardim D, Via G, Czosnyka M, et al. Brain ultrasonography: methodology, basic and advanced principles and clinical applications. A narrative review. Intensive Care Med. 2019;45:913-27.

3. Ract C, Le Moigno S, Bruder N, Vigué B. Transcranial Doppler ultrasound goal-directed therapy for the early management of severe traumatic brain injury. Intensive Care Med. 2007;33(4):645-51 Epub 2007 Feb 27.

4. Robba C, Cardim D, Tajsic T, Pietersen J, Bulman M, Donnelly J, et al. Ultrasound non-invasive measurement of intracranial pressure in neurointensive care: a prospective observational study. PLoS Med. 2017;14: e1002356.

5. Rasulo FA, Bertuetti R, Robba C, Lusenti F, Cantoni A, Bernini M, et al. The accuracy of transcranial Doppler in excluding intracranial hypertension 
following acute brain injury: a multicenter prospective pilot study. Crit Care. 2017;21:44.

6. Robba C, Donnelly J, Bertuetti R, Cardim D, Sekhon MS, Aries M, et al. Doppler non-invasive monitoring of ICP in an animal model of acute intracranial hypertension. Neurocrit Care. 2015;23:419-26.

7. Consensus Group on Transcranial Doppler in Diagnosis of Brain Death. Latin American consensus on the use of transcranial Doppler in the diagnosis of brain death. Rev Bras Ter Intensiva. 2014;26(3):240-52.

8. Kuo J, Chen C, Chio C, Chang C, Wang C, Yang C, et al. Time dependent validity in the diagnosis of brain death using transcranial Doppler sonography. J Neurol Neurosurg Psychiatry. 2006;77:646-9.

9. Donnelly J, Aries MJ, Czosnyka M. Further understanding of cerebral autoregulation at the bedside: possible implications for future therapy. Expert Rev Neurother. 2015;15:169-85.

10. Panerai RB. Transcranial Doppler for evaluation of cerebral autoregulation. Clin Auton Res. 2009;19:197-211.

11. Czosnyka M, Smielewski P, Kirkpatrick P, Menon DK, Pickard JD. Monitoring of cerebral autoregulation in head-injured patients. Stroke. 1996;27:1829-34.

12. Diringer MN, Bleck TP, Claude Hemphill J, Menon D, Shutter L, Vespa P, et al.

Critical care management of patients following aneurysmal subarachnoid hemorrhage: recommendations from the Neurocritical Care Society's Multidisciplinary Consensus Conference. Neurocrit Care. 2011;15:211-40.

13. Kumar G, Shahripour RB, Harrigan MR. Vasospasm on transcranial Doppler is predictive of delayed cerebral ischemia in aneurysmal subarachnoid hemorrhage: a systematic review and meta-analysis. J Neurosurg. 2016;124:1257-64.

14. Vora Y, Suarez-Almazor M, Steinke D, Martin M, Findlay J. Role of transcranial Doppler monitoring in the diagnosis of cerebral vasospasm after subarachnoid hemorrhage. Neurosurgery. 1999;44:1237-47-8.

15. Lindegaard KF, Nornes H, Bakke SJ, Sorteberg W, Nakstad P. Cerebral vasospasm diagnosis by means of angiography and blood velocity measurements. Acta Neurochir. 1989;100:12-24.

\section{Publisher's Note}

Springer Nature remains neutral with regard to jurisdictional claims in published maps and institutional affiliations. 